\title{
Contrastive salient alternatives: focus on bound pronouns*
}

\author{
Clemens Mayr \\ Zentrum für Allgemeine \\ Sprachwissenschaft
}

\begin{abstract}
The presence of contrastive focus on pronouns interpreted as bound variables is puzzling because such variables do not refer. Therefore it is unclear how two bound variables can be made to contrast with each other. It is argued that previous approaches to this puzzle face an empirical problem. The account offered is based on the idea that the alternatives introduced by focused bound pronouns denote individuals. Introducing the novel concept of salient alternatives, it is shown that this allows one to get bound pronouns to contrast. Furthermore a modification of Rooth's (1992) -operator is suggested: Contrastiveness is a requirement of the operator, which is modelled as a definedness condition.
\end{abstract}

Keywords: focus, bound variables, alternatives, contrastiveness

\section{Introduction}

It is generally accepted that a focus-mark (F-mark) on a syntactic constituent can be used to contrast that constituent with another one, usually preceding it. In pretheoretical terms, if a constituent is contrastively focused there must be an antecedent constituent that can serve as an alternative to it and moreover is non-identical to it. Sauerland (1998, 2000, 2008) (also cf. Dimitriadis 2001 and Jacobson 2000) first addresses the question how this is compatible with contrastive focus on pronouns under the bound variable reading, such as in (1-a). ${ }^{1}$ As such pronouns do not have a denotation, it becomes unclear how the focused pronoun can be made to contrast with the antecedent pronoun. Moreover, we notice that the F-mark on the pronoun

* I wish to thank Sigrid Beck, Noam Chomsky, Amy Rose Deal, Klaus von Heusinger, Jim Huang, Peter Jenks, Hans Kamp, Hiroki Narita, Viola Schmitt, Giorgios Spathas, Satoshi Tomioka, and Hedde Zeijlstra for discussion and comments. I especially thank Gennaro Chierchia and Irene Heim for many invaluable suggestions and helpful comments on a longer, and more detailed version of the paper. Some comments of the people mentioned unfortunately only made into that version for reasons of space. Moreover, I thank the audiences at Harvard, at GLOW 33 in Wrocław, and at SALT 20 in Vancouver.

1 Throughout capitals indicate stress, i.e., an F-marked constituent. 
is not obligatory (1-b), which further complicates things. As known since at least Schwarzschild 1999, F-marks are usually not optional.

(1) a. Every student cut his (own) arm, and every TEACHER cut HIS arm

b. Every student cut his (own) arm, and every TEACHER cut his arm

The present paper shows that previous analyses of focus on bound pronouns face empirical complications. I then suggest a new account based on Rooth's (1985) approach to focus in terms of a two-dimensional semantics - that is, focus introduces a set of alternative denotations. The alternatives of a (bound) pronoun is taken to be a set of individuals. Following Rooth (1992), I assume that focus is interpreted by the so-called $\sim$-operator, which requires that there is an antecedent constituent whose denotation is a member of the alternatives contributed by focus. Introducing the novel concepts of salient alternative and compositional reconstruction, I argue that the first sentence in (1-a) indeed leads to alternatives with individuals standing in for the pronoun and moreover contrasting with the ordinary meaning of the relevant constituent in the second sentence. The antecedent sentence provides the correct types of alternatives for focus licensing if it is allowed that they are compositionally reconstructed with the help of the denotation of the antecedent constituent and other material in the context. In case there is an F-mark on a bound pronoun, there must be a -operator embedded somewhere in the scope of the quantifier. The semantic contribution of that F-mark thereby becomes invisible for further, higher focus evaluation. This has the consequence that the antecedent constituent relevant for compositional reconstruction cannot be bigger than the VP in the antecedent sentence. I will moreover argue for a modification of the $\sim$-operator which directly encodes the contrastiveness requirement in its meaning. Together with the assumption that (1-a) has an embedded -operator, this has the consequence that focus on the bound pronoun becomes obligatory if that syntactic option is chosen. If there is no embedded $\sim$-operator, focus must be absent.

The paper is structured as follows: In section 2, I discuss the puzzle raised by focus on bound pronouns in more detail. The empirical challenge for previous approaches is also introduced. Section 3 presents the new account. Section 4 discusses two predictions of the account and a potential problem. The last section concludes the paper.

\section{The problem caused by stressed bound pronouns}

I now discuss the problem raised by focus on bound pronouns in more detail. The problem is sketched making use of Rooth's (1992) theory of focus licensing, which 
Contrastive salient alternatives

is itself based on the theory of focus interpretation in Rooth $1985 .^{2}$

\subsection{Rooth's theory of focus licensing}

According to Rooth's theory, F-marks contribute a second semantic value to the interpretive procedure, namely a focus value. The ordinary value is the usual one ignoring the semantic content of the F-mark. The focus value is the set of alternative meanings of the same type as the ordinary meaning: ${ }^{3}$

\section{Semantic values}
a. (i) $\quad\left[\left[\mathrm{A}_{F, \sigma}\right]\right]^{g}=A$
(ii) $\left[\left[\mathrm{A}_{F, \sigma}\right]\right]^{f}=D_{\sigma}$
b. (i) $\left[\left[\mathrm{A}_{\sigma}\right]\right]^{g}=A$
(ii) $\left[\left[\mathrm{A}_{\sigma}\right]\right]^{f}=\left\{\left[\left[\mathrm{A}_{\sigma}\right]\right]^{g}\right\}$

How is focus licensed in this theory? For a discourse like (3) the LFs in (4) are assumed. Note that the focus utterance in (4-b) has the so-called -operator adjoined, which is co-indexed with (4-a), which means that A's utterance functions as the antecedent for the focus utterance.
A: Sue married John?
B: No, RITA married John
a. [IP Sue married John $]_{1}$
b. $\quad\left[\mathrm{IP}_{2} \sim_{1} \mathrm{C}\left[\mathrm{IP}_{1} \mathrm{Rita}_{F}\right.\right.$ married John $\left.]\right]$

2 For reasons of space other approaches to focus licensing cannot be addressed. As I show in Mayr 2010 a parallel problem arises for instance in Schwarzschild's (1999) theory which does not use a second interpretation value. There I argue that an operator interpreting foci and a second interpretation value are essential for dealing with focus on bound pronouns.

3 In addition to the normal rules of functional application and predicate abstraction, we need appropriate rules for the focus values. Following Hamblin (1973) and Rooth (1985) the former is defined as point-wise application:

(i) Given branching node A with daughters B of type $\langle\sigma, \tau\rangle$ and $\mathrm{C}$ of type $\sigma,[[\mathrm{A}]]^{f}=\{f(x) \in$ $D_{\tau}: f \in[[\mathrm{B}]]^{f}$ and $\left.x \in[[\mathrm{C}]]^{f}\right\}$.

The rule of predicate abstraction should deliver an object of type $\langle\langle e, s t\rangle, t\rangle-$ that is, a set of properties - when dealing with focus values. The simplified rule in (ii) does so by letting the F-mark introduce a variable in the modified assignment and existentially quantifying over it.

(ii) If $\mathrm{A}$ is a branching node with daughters $\mathrm{B}$ of type $\tau$ and a numerical index i, $\llbracket \mathrm{A}]]^{f}=\{f \in$ $D_{\langle e, \tau\rangle}: \exists y\left[f=\lambda x \cdot\left[[\mathrm{B}]^{g[x / i],[y / F]}\right]\right\}$ 
The $\sim$-operator takes two arguments, a contextual restriction $g(C)$ and the ordinary value of the constituent it is attached to. It is defined as in (5). It says that the denotation of $g(C)$ must be a subset of the focus value of the sister of $\sim$. Moreover, the focus value is reset to the ordinary value.

$$
\text { a. } \quad \begin{aligned}
& {\left[\left[\left[X \sim \mathrm{C}\left[\begin{array}{lll}
Y & \ldots
\end{array}\right]\right]\right]\right]^{g}=} {\left[\left[\left[\begin{array}{ll}
Y & \ldots
\end{array}\right]\right]^{g}\right.} \\
& \text { if } g(C) \subseteq\left[\left[\left[\begin{array}{lll}
Y & \ldots
\end{array}\right]\right]^{f},\right. \text { otherwise undefined } \\
& \text { b. } \quad\left[\left[\left[X \sim \mathrm{C}\left[\begin{array}{lll}
Y & \ldots
\end{array}\right]\right]\right]^{f}=\left\{\left[\left[\left[\begin{array}{lll}
Y & \ldots
\end{array}\right]\right]^{g}\right\}\right.\right.
\end{aligned}
$$

For (3) this means the following. Coindexation has the consequence that $g(C)$ is set to the ordinary value of (4-a). If that value is a subset of the focus value of the constituent that $\sim$ is attached to, $\mathrm{IP}_{1}$, then the F-mark is licensed. The ordinary value of (4-a) is the proposition that Sue married John. The focus value for $\mathrm{IP}_{1}$ in (4-b) is the set of alternatives to the proposition that Rita married John, where Rita is replaced by alternative individuals (6). The ordinary value of the antecedent is a subset/member of (6). Thus focus on Rita is licensed.

$$
\left[\left[\mathrm{IP}_{1}\right]\right]^{f}=\left\{\lambda \text { w.marry }_{w}(x, \text { John }) \mid x \in D_{e}\right\}
$$

\subsection{The problem}

\subsubsection{Unlicensed focus}

For the initial example repeated as (7), an approach parallel to the one just sketched would assume the LFs in (8).

(7) Every student cut his (own) arm, and every TEACHER cut HIS arm

$$
\text { a. [IP every student } \left.1\left[\mathrm{t}_{1} \text { cut } 1 \text { 's arm }\right]\right]_{2}
$$

b. $\left[\mathrm{IP}_{2} \sim_{2} \mathrm{C}\left[\mathrm{IP}_{1}\right.\right.$ every teacher $F$ $3\left[\mathrm{t}_{3}\right.$ cut $3_{F}$ 's arm $\left.\left.]\right]\right]$

The alternatives of a bound pronoun are of type $e$. Therefore the focus value of $\mathrm{IP}_{1}$ in (8-b) is as in (9) - that is, it corresponds to the set of propositions \{ that every boy cut John's arm, that every teacher cut Mary's arm,... \}, where the bound pronoun is replaced with individual-denoting expressions.

$$
\left.\left[\mathrm{IP}_{1}\right]\right]^{f}=\left\{\lambda w . \forall x\left[P_{w}(x) \rightarrow \operatorname{cut}_{w}\left(x, y^{\prime} s \text { arm }\right)\right] \mid y \in D_{e}, P \in D_{\langle e, s t\rangle}\right\}
$$

The ordinary value of (8-a) corresponds to the proposition that every student cut his own arm. This proposition, however, is not a member/subset of (9). Thus the focus on the bound pronoun is not licensed according to Rooth's theory, and (7) should be ungrammatical. The reason for this is that a bound variable is not a member of its own alternatives, the consequence of which is that the bound variable reading - i.e., 
the ordinary value of (8-b) - cannot be recovered from the alternatives in (9). ${ }^{4}$

As noticed by Jacobson (2000) and Sauerland (2000), it cannot be claimed that contrastive stress on bound pronouns is licensed because of an assignment function $g$ that assigns differing values to the antecedent variable and the focused variable. That is, it is not enough to adjoin the $\sim$-operator below the binder in (8-b), say to the DP $\left[3_{F}\right.$ 's arm], and moreover require that $g(1) \neq g(3)$. In this case, the ordinary value of the antecedent DP would be a member of the focus value of the focus DP. Contrastiveness would hold as well. The reason this approach does not work is (10). In case the domains of the quantifiers overlap, focus on the bound pronoun is prohibited. If all that were required were that the assignments to the variables involved differ, it would be predicted that focus should be licensed, however. It seems that focus is impossible in (10) because due to the incomplete contrastiveness of the quantificational domains, the bound pronouns do not fully contrast either. I.e., focus on a bound pronoun is only licensed if it is also licensed when the binder is taken into account.

*I expected every student to call his father, but only every YOUNG student called HIS father.

(Sauerland 1998:206)

\subsubsection{Optionality}

As evidenced by (1), focus on the bound pronoun is optional. But Schwarzschild (1999) has shown that in all other cases, focus is either obligatorily present or obligatorily absent. Looking at A's utterance, we notice that only B and C are felicitous replies. The reason is that Sue contrasts with Mary and therefore must be stressed. Mary, on the other hand, does not contrast with Mary and must not be stressed. It follows that if contrastiveness holds, focus must be used. If it does not hold, focus must not be used. Schwarzschild terms this principle AvoidF, which strives to reduce F-marks whenever possible.

$$
\begin{array}{ll}
\text { A: } & \text { John kissed Mary } \\
\text { B: } & \text { Yes. And, BILL kissed SUE }
\end{array}
$$

4 Note that on the basis of (i) it cannot be claimed that the stress on the bound pronoun is merely inherited from the stress on the restrictor of the quantifier via some syntactic agreement mechanism (also cf. Jacobson 2000 and Sauerland 2000). In (i) the quantifier does not have anything to contrast with and is therefore not stressed. Nevertheless the bound pronoun can bear an F-mark.

$\begin{array}{ll}\text { (i) } & \text { Every student cut John's arm } \\ \text { B: } & \text { No, every student cut HIS arm }\end{array}$ 


$$
\begin{aligned}
& \text { B': \#Yes. And, BILL kissed Sue } \\
& \text { C: Yes. And, BILL kissed Mary (too) } \\
& \text { C': \#Yes. And, BILL kissed MARY (too) }
\end{aligned}
$$

If there is such a principle that dictates focus to be present whenever possible, it becomes a mystery why focus is optional in (1). That is, at the current stage even if one finds a way to address the issue discussed in the preceding subsection, the principle that reduces foci whenever possible would rule out (7). The reason is that there is a more optimal way of uttering the sentence in (1-a), namely without stress on the bound pronoun as in $(1-b) .^{5}$

\subsubsection{Why a functional analysis fails}

Sauerland $(2000,2008)$ assumes that the sentences in (1) have differing LFs. ${ }^{6}$ The one with focus on the bound pronoun has the LFs in (12), whereas the one without has the LFs in (13). That is, Sauerland proposes that the bound pronoun must be an E-type pronoun corresponding to a definite description when it is focused. Notice that it is the property in the definite description that bears the F-mark. ${ }^{7}$

a. [every student $]_{9}\left[1\left[\mathrm{t}_{1} \text { cut }\left[\left[\text { the }_{1} \text { student }_{4}\right] \text { 's arm }\right]\right]\right]_{8}$

b. $\quad\left[\sim_{9} \mathrm{C}_{2}\right.$ [every teacher $\left.\left.{ }_{5, F}\right]\right]\left[\sim_{8} \mathrm{C}_{1} 1\left[\mathrm{t}_{1}\right.\right.$ cut [[the ${ }_{1}$ teacher $\left._{5, F}\right]$ 's arm] ]]

a. [every student $1\left[\mathrm{t}_{1}\right.$ cut 1 's arm $\left.]\right]_{2}$

b. $\quad\left[\sim_{2} \mathrm{C}\right.$ [every teacher $F_{F} 3\left[\mathrm{t}_{3}\right.$ cut 3 's arm] $\left.]\right]$

For reasons of space, I will keep the discussion informal. Assuming that the property embedded in the definite description makes the VPs partial functions, the denotations in (14) obtain for the VPs in (12). The first VP is only defined for students, whereas

5 It is easy to see that (1-b) is licensed by the theory of focus licensing sketched. The antecedent sentence denotes the proposition that every student cut his own arm. The focus value of the focus sentence is the set of propositions \{that every student cut his own arm, that every teacher cut his own arm,... $\}$. The former is a member of the latter. Contrastiveness also holds. I.e., the requirements of $\sim$ are satisfied.

6 Also cf. Elbourne 2005, who follows Sauerland to a large extent. The proposal offered by Jacobson (2000) is similar to the one by Sauerland, although couched in a variable-free semantics. Crucially, though, in her account the contrasting pronouns used are assumed to denote the identity function over different contrasting domains. This would also run into the problem discussed below. See Mayr 2010 for detailed discussion.

7 In other words, the NP in the restrictor of the quantifier functions as the syntactic antecedent for the NP in the pronoun indicated by coindexation (cf. the discussion in Heim 1990 and Chierchia 1990: 158f. especially). This could, for instance, be done by treating pronouns as cases of ellipsis (cf. Heim 1990, Elbourne 2005 a.o.). Sauerland (2008) himself argues against an ellipsis analysis, but this is immaterial to the present discussion. For simplicity, I will present the semantic content of the NP inside the pronoun syntactically. 
Contrastive salient alternatives

the second is only defined for teachers.

$$
\begin{array}{ll}
\text { a. } & \lambda x . \lambda w: \operatorname{student}_{w}(x) \cdot \operatorname{cut}_{w}\left(x, x^{\prime} \text { sarm }\right) \\
\text { b. } & \lambda x \cdot \lambda w: \operatorname{teacher}_{w}(x) \cdot \operatorname{cut}_{w}\left(x, x^{\prime} \text { s arm }\right)
\end{array}
$$

The focus value for the VP in (12-b) is as in (15), where the property of being a teacher is replaced with its alternatives. (14-a) is a member of that set. Moreover the partial functions in (14) contrast with each other. Therefore the focus on the pronoun is licensed. It is easy to see that the focus on the restrictor in the quantifier is also licensed.

$$
[[\mathrm{VP}]]^{f}=\left\{\lambda x . \lambda w: Q_{w}(x) . c_{w}\left(x, x^{\prime} s \text { arm }\right) \mid Q \in D_{\langle e, s t\rangle}\right\}
$$

It must be noted that under this analysis the focus on the bound pronoun cannot be dropped. If one were to do so, the focus value of the VP in (12-b) would correspond to the singleton containing just (14-b). In other words, (14-a) would not be a member of the focus value, and thus the structure would not be licensed. The principle striving to reduce F-marks cannot apply, as only the structure with F-mark is licensed to begin with. As already said, the construction without focus on the bound pronoun is licensed by LFs with plain variables (13). We have already seen how licensing proceeds in this case. Summarizing, Sauerland's account explains the presence of focus on bound pronouns and the perceived optionality.

There is a problem with this account, and it has to do with the possible cooccurrence of an F-mark on the bound pronoun and additive too:

(16) Every director discussed his film, and every PRODUCER discussed HIS film, too

Let us assume the anaphoric entry for too in (17) following Heim (1992: 189). That is, too associates with focus on $\mathrm{X}$ and requires that there is a contrasting alternative $\mathrm{Y}$ in the context such that when $\mathrm{X}$ is replaced by $\mathrm{Y}$, the denotation of the outcome is true.

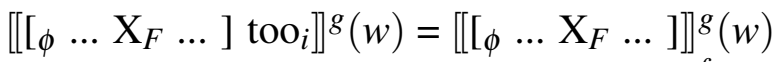

$$
\begin{aligned}
& \text { if }\left[\left[\mathrm{Y}_{i}\right]\right]^{g} \in[[\mathrm{X}]]^{f},\left[\left[\mathrm{Y}_{i}\right]\right]^{g} \neq[[\mathrm{X}]]^{g} \text { and } \\
& {\left[\left[\left[\begin{array}{lll}
\phi & \ldots & Y_{i} \ldots
\end{array}\right]\right]^{g}(w)=1\right. \text {, otherwise undefined }}
\end{aligned}
$$

The LFs for (16) would accordingly be as in (18). The problem with (18) is that when the restrictor producer is replaced by director, as the semantics of too would have it, the value of the resulting sentence is undefined. The reason is that the predicate denoted by the VP is only defined for producers, i.e., it is undefined for directors. Thus (16) should be ungrammatical. 


$$
\begin{aligned}
& \text { a. every director }{ }_{5} 1 \text { [ } \mathrm{t}_{1} \text { discussed [[the }{ }_{1} \text { director]'s film]] } \\
& \text { b. every } \left.\operatorname{producer}_{F} 1\left[\mathrm{t}_{1} \text { discussed [[the }{ }_{1} \operatorname{producer}_{F}\right] \text { 's film] }\right] \text { too } 5
\end{aligned}
$$

The account cannot be saved by assuming that too associates with both foci so that both instances of producer are replaced by director. The reason for this is that too does not associate with two foci. This fact has already been noticed by Kaplan (1984):

$$
\begin{aligned}
& \text { a. *Jo had fish and Mo had soup too. } \\
& \text { b. Jo had fish and Mo had soup. } \\
& \text { (Kaplan 1984:510) }
\end{aligned}
$$

Thus the attractive account making use of underlying functions makes the wrong predictions for certain cases. As already noted in footnote 6 above, the problem carries over to the account offered by Jacobson (2000). In the following section I will suggest an alternative analysis.

\section{The account}

The present proposal consists of two modifications of Rooth's (1992) theory of focus licensing. I will discuss each one in turn. But before doing so, I want to suggest the following: Consider the example in (20) and assume that the corresponding LF is the one in (21). There are two -operators: one is attached to the sentence and one to the VP including the binder. ${ }^{8}$

(20) Every director discussed his film, and every ACTOR discussed HIS film

$$
\left[\sim \mathrm { C } _ { 2 } \left[\mathrm{IP}[\text { every actor } F]\left[\mathrm{VP}_{2} \sim \mathrm{C}_{1}\left[\mathrm{VP}_{1} 1\left[\mathrm{t}_{1} \text { discussed } 1_{F} \text { 's film] }\right]\right]\right]\right.\right.
$$

The focus values of $\mathrm{VP}_{1}$ and IP are given in (22-a) and (22-b), respectively. It is important to notice that the focus contribution by the F-mark on the bound pronoun is annihilated by the embedded -operator - that is, the focus value of IP only has a focus variable contributed by the F-mark on the restrictor. Remember that the $\sim$-operator resets the focus value of the constituent dominating it to the singleton corresponding to the ordinary value of its sister constituent. This has the effect that the denotation of the first sentence in (20) can serve as the antecedent for the second sentence as a whole, as it is a member of (22-b).

$$
\text { a. } \left.\left.\quad\left[\mathrm{VP}_{1}\right]\right]^{f}=\left\{\lambda x . \lambda \text { w.discuss }_{w}\left(x, y^{\prime} \text { s film }\right)\right] \mid y \in D_{e}\right\}
$$

8 Other options which I will not discuss here for reasons of space are possible. What is crucial is that there is one $\sim$-operator per F-mark and moreover that one of them is embedded in the scope of the quantifier. The reader can verify that these LFs will lead to essentially the same results as the LF discussed in the text. 
Contrastive salient alternatives

$$
\text { b. } \quad[[\mathrm{IP}]]^{f}=\left\{\lambda w . \forall x\left[P_{w}(x) \rightarrow \operatorname{discuss}_{w}\left(x, x^{\prime} \text { s film }\right) \mid P \in D_{\langle e, s t\rangle}\right\}\right.
$$

This solves part of the problem discussed in section 2.2.1: the requirements of the higher $\sim$-operator are satisfied, i.e., the F-mark on the restrictor is licensed. The question why the F-mark on the bound pronoun is licensed still remains. Considering the focus value of $\mathrm{VP}_{1}$ corresponding to the set of properties \{discuss John's film, discuss Mary's film, ... \}, we notice that the requirement of the lower $\sim$-operator cannot be fulfilled. The reason is that the denotation of the antecedent VP is not a member of (22-a), as it involves variable-binding. ${ }^{9}$

Let us turn to the solution proposed for this problem.

\subsection{Formal vs. salient alternatives}

Following Rooth I assume that the $\sim$-operator requires that the contextual restriction $g(C)$ be a subset of the focus value of the sister constituent of $\sim$. I will refer to the alternatives corresponding to the focus value as the set of formal alternatives being activated by $\sim$. The context must now provide salient alternatives that match the formal ones in their form. The salient alternatives are provided by the linguistic object present in the context with which the $\sim$-operator is coindexed with. In contrast to Rooth's analysis, however, I allow for the possibility of having salient alternatives be compositionally reconstructed from the linguistic object in question and other information that is part of the context. The term compositional reconstruction is meant to refer to the process of applying the denotation of the antecedent linguistic object to material provided by the context - that is, other linguistic objects in the context - or vice versa. That is, salient alternatives are defined as follows: ${ }^{10}$

\section{Salient alternatives}

An alternative is salient if it corresponds to the ordinary value of a linguistic object $\mathrm{X}$ in the context, $\left[[\mathrm{X}]^{g}\right.$, or it can be compositionally reconstructed using $[[\mathrm{X}]]^{g}$.

I moreover require that salient alternatives matching the formal ones must be used. In other words, the salient alternatives that satisfy the requirements posed by the activated alternatives must be set as identical to the denotation of the contextual restriction $\mathrm{C}$. I suggest that this requirement is directly encoded in the meaning of the $\sim$-operator (here $A_{S}$ stands for salient alternative):

9 Note that the problem cannot be simply circumvented by claiming that the actual antecedent VP does not involve the binder. This would, again, predict that focus on a bound pronoun is even licensed when the domains of the quantifiers overlap. Recall (10) above.

10 In addition, it should be possible to have alternatives count as salient that can be inferred by contextual entailment or implicature. 


$$
\text { a. } \begin{aligned}
{\left[\left[\left[X \sim \mathrm{C}\left[\begin{array}{lll}
Y & \ldots
\end{array}\right]\right]\right]\right]^{g}=} & {\left[\left[\left[\begin{array}{ll}
Y & \ldots
\end{array}\right]\right]^{g}\right.} \\
& \text { if } g(C) \subseteq\left[\left[\left[\begin{array}{lll}
Y & \ldots
\end{array}\right]\right]^{f},\right. \text { and } \\
& \forall A_{S}\left[A_{S} \in\left[\left[\left[\begin{array}{lll}
Y & \ldots
\end{array}\right]\right]^{f} \rightarrow A_{S} \in g(C)\right],\right. \text { otherwise } \\
& \text { undefined }
\end{aligned}
$$

b. $\quad\left[\left[\left[X \sim \mathrm{C}\left[\begin{array}{lll}Y & \ldots\end{array}\right]\right]\right]\right]^{f}=\left\{\left[\left[\left[Y Y^{\prime} \ldots\right]\right]\right]^{g}\right\}$

Recall that the denotation of the antecedent VP in (20) does not match the activated formal alternatives, as the former involves variable binding whereas the latter don't. What does the process of compositional reconstruction of salient alternatives do for us when presented with this problem? Assume the following situation: $a, b, c$ are the relevant directors in the context. Each of them discussed his own film. Applying the directors point-wise to the denotation of the antecedent VP, (25-a), results in the set of propositions in (25-b). The latter is equivalent to the set in (25-c). Here the subjects are scoped out.

$$
\begin{aligned}
& \text { a. }\left\{\lambda x \cdot \lambda w \cdot \operatorname{discuss}_{w}\left(x, x^{\prime} \text { s film }\right)\right\}\left(\left\{\begin{array}{l}
a \\
b \\
c
\end{array}\right\}\right) \\
& \text { b. }=\left\{\begin{array}{l}
\lambda w \cdot \operatorname{discuss}_{w}\left(a, a^{\prime} \text { s film }\right) \\
\lambda w \cdot \operatorname{discuss}_{w}\left(b, b^{\prime} \text { s film }\right) \\
\lambda w \cdot \operatorname{discuss}_{w}\left(c, c^{\prime} s \text { film }\right)
\end{array}\right\} \\
& \text { c. }=\left\{\begin{array}{l}
\lambda x \cdot \lambda w\left[\operatorname{discuss}_{w}\left(x, a^{\prime} s \text { film }\right)\right](a) \\
\lambda x \cdot \lambda w\left[\operatorname{discuss}_{w}\left(x, b^{\prime} s \text { film }\right)\right](b) \\
\lambda x \cdot \lambda w\left[\operatorname{discuss}_{w}\left(x, c^{\prime} s \text { film }\right)\right](c)
\end{array}\right\}
\end{aligned}
$$

But notice that by functional application we have derived a set of predicates that matches the activated formal alternatives if we ignore the scoped subjects in (25-c), namely (26). According to our new entry for the $\sim$-operator, (24), this means that (26) must be the set of salient alternatives used, i.e., (26) corresponds to the denotation of the contextual restriction $\mathrm{C}$ used by $\sim$. It thus follows that in our slightly modified Roothian system, focus on bound pronouns is actually licensed if there is a $\sim$-operator embedded in the scope of the quantifier.

$$
\left\{\begin{array}{l}
\lambda x . \lambda w \cdot \operatorname{discuss}_{w}\left(x, a^{\prime} s \text { film }\right) \\
\lambda x . \lambda w . \operatorname{discuss}\left(x, b^{\prime} s \text { film }\right) \\
\lambda x . \lambda w . \operatorname{discus} s_{w}\left(x, c^{\prime} s \text { film }\right)
\end{array}\right\}
$$

The obvious next question is why F-marks on bound pronouns are optional. This question can be rephrased as follows: Why does the principle that strives to reduce foci not rule out the presence of the F-mark on the bound pronoun? One would expect the sentence without F-mark to be more optimal than the one with F-mark 
in case the former is licensed. And we already know that it is licensed. In the following, I suggest that once the issue of contrastiveness is addressed in more detail, the problem dissolves.

\subsection{Contrastiveness ${ }^{11}$}

Assume that the second sentence in (27-a) corresponds to the LF in (28-a) (which is identical to the one discussed in the preceding subsection) and the one in (27-b) to the LF in (28-b). We already know that both structures are licensed by our theory of focus licensing: the former because of the modification just introduced, and the latter because there is no F-mark on the bound pronoun.

a. Every director discussed his film, and every ACTOR discussed HIS film

b. Every director discussed his film, and every ACTOR discussed his film

a. $\left[\sim \mathrm{C}_{2}\left[\mathrm{IP}[\right.\right.$ every actor $F]\left[\mathrm{vP}_{2} \sim \mathrm{C}_{1}\left[\mathrm{vP}_{1} 1\left[\mathrm{t}_{1}\right.\right.\right.$ discussed $1_{F}{ }^{\prime}$ s film $\left.\left.\left.]\right]\right]\right]$

b. $\left[\sim \mathrm{C}\left[\mathrm{IP}\left[\right.\right.\right.$ every actor $\left._{F}\right]\left[\mathrm{VP} 1\left[\mathrm{t}_{1}\right.\right.$ discussed 1 's film $\left.\left.\left.]\right]\right]\right]$

For a principle like AvoidF that reduces F-marks to make sense at all, it needs to compare structures that are not too different. Let us think of this as meaning that the structures differ at most in the number of F-marks present. Notice now that viewed in this way, (28-b) cannot be a competitor of (28-a). In addition to differing with respect to the number of F-marks, the two also differ in the number of $\sim$-operators. It follows - if (28-a) does not have a competitor where there is no F-mark on the bound pronoun and that is licensed by the focus licensing principle - that (27-a) is licensed by our theory. ${ }^{12}$ I therefore suggest that the $\sim$-operator has contrastiveness built into it so that we end up with a -operator for contrastive uses. The way this is done is through a presupposition requiring that the ordinary value of the sister of $\sim$ is not a member of the denotation of the contextual restriction $\mathrm{C}$. In other words, none of the salient alternatives making up $g(C)$ must be equivalent to the ordinary value of the sister of $\sim$. Assume that the $\sim$-operator, just like Rooth's (1985) only, is defined cross-categorially. The final meaning of the $\sim$-operator attached to predicate-denoting constituents is then as in (29) where Heim \& Kratzer's (1998) convention is used for indicating presuppositions.

$$
\text { a. } \begin{aligned}
{\left[\left[\left[X \sim \mathrm{C}\left[\begin{array}{lll}
Y & \ldots
\end{array}\right]\right]\right]^{g}=\right.} & \lambda x: g(C) \subseteq\left[\left[\left[\begin{array}{ll}
Y & \ldots
\end{array}\right]\right]^{f} \wedge\right. \\
& \forall A_{S}\left[A_{S} \in[[[Y \ldots]]]^{f} \rightarrow A_{S} \in g(C)\right] \wedge \\
& {\left[\left[\left[\begin{array}{lll}
Y & \ldots
\end{array}\right]\right]^{g}(x) \notin\{g(C)\}(x) \cdot\left[\left[\begin{array}{lll}
g & \ldots
\end{array}\right]\right]^{g}(x)\right.}
\end{aligned}
$$

11 Thanks to Irene Heim (p.c.) for helping me with this part.

12 We already know that (27-b) is grammatical anyway because adding an F-mark to the pronoun in the corresponding LF in (28-b) leads to an unlicensed focus. 


\section{b. $\quad\left[\left[\left[X \sim \mathrm{C}\left[\begin{array}{ll}Y & \ldots\end{array}\right]\right]\right]^{f}=\left\{[[[Y, \ldots]]]^{g}\right\}\right.$}

Concentrating only on the contrastiveness presupposition, (29) has the following consequence for (28-a): Were the F-mark on the bound pronoun left out, it would follow that the focus value of $\mathrm{VP}_{1}$ would correspond to the singleton containing just the ordinary value of $\mathrm{VP}_{1}$. From this it moreover follows that the alternatives made salient by the context that make up $g(C)$ are members of that set. The denotation of the antecedent VP provides just such an alternative, as it is identical to the denotation of $\mathrm{VP}_{1}$. But now the requirement introduced by the $\sim$-operator is contradictory as it requires that the ordinary value of $\mathrm{VP}_{1}$ not be in $g(C)$. Due to the reasoning just described, however, it is the only member of $g(C)$. I.e., the requirement added by $\sim$ can never be fulfilled if there is no F-mark on the bound pronoun. In other words, the contrastiveness condition requires the F-mark on the bound pronoun if a -operator is present in the scope of the quantifier. This is just what we want.

To sum up: The present theory accounts for why focus on bound pronouns is possible at all. I proposed that there must be a -operator located in the scope of the quantifier whenever there is an F-mark on a bound pronoun. I argued for the process of compositional reconstruction to generate salient alternatives that match the formal ones activated by the $\sim$-operator. This makes it possible to license focus on bound variables in principle. I.e., it solves the first problem discussed in the preceding section. Moreover, I argued for a contrastiveness requirement introduced by the $\sim$-operator that has the consequence that F-marks on bound pronouns become obligatory as soon as there is a $\sim$-operator in the scope of the quantifier. The optionality of focus has been accounted for by employing two different syntactic structures for the relevant constructions.

\section{Some consequences}

Let us now turn to some consequences of the analysis argued for in the preceding section.

\subsection{Overlapping domains}

Recall that bound pronouns cannot be stressed if the domains of the quantifiers involved overlap. This is necessarily the case in (30), repeated from above, where the set of young students is a subset of the set of all students.

(30) *I expected every student to call his father, but only every YOUNG student called HIS father.

(Sauerland 1998:206) 
Let us see how the present account explains (30). In the account argued for in the preceding section it must be the case that there is a $\sim$-operator embedded in the scope of the quantifier interpreting the contribution of the F-mark on the bound pronoun. Moreover, I suggested that the $\sim$-operator incorporates contrastiveness directly. In particular, it requires that the ordinary value of the constituent it is attached to is not a member of $g(C)$. Thus, the denotation of the VP-constituent for the second sentence in (30) must be as follows.

$$
\begin{aligned}
{[[\mathrm{VP}]]^{g}=} & \lambda x \cdot \lambda w: \lambda y \cdot \lambda w^{\prime}\left[\operatorname{call}_{w^{\prime}}\left(y, y^{\prime} \text { s father }\right)\right](x) \notin\{g(C)\}(x) . \\
& \operatorname{call}_{w}\left(x, x^{\prime} \text { s father }\right)
\end{aligned}
$$

(31) is a partial function. But what exactly does this partiality express? In order to know whether the proposition $\lambda y \cdot \lambda w^{\prime}\left[\operatorname{call}_{w^{\prime}}\left(y, y^{\prime} s\right.\right.$ father $\left.)\right](x)$ is truly not a member of $\{g(C)\}(x)$, we must first determine what the denotation of $g(C)$ is. The salient alternatives matching the formal ones must be predicates of the form $\lambda x . \lambda w_{\text {.call }}\left(x, a^{\prime}\right.$ father $)$, where $a$ denotes a student. $g(C)$ is set as identical to these alternatives. Assume the following situation: John, Bill, Harold, and Francis are the relevant students. Out of these only Harold and Francis qualify as young. Moreover, assume that I expected each of them to call his own father. In that situation $g(C)$ is as in (32).

$$
g(C)=\left\{\begin{array}{c}
\lambda x \cdot \lambda w_{\text {call }}\left(x, \text { John' }^{\prime} \text { f father }\right) \\
\lambda x \cdot \lambda w \cdot \text { call }_{w}\left(x, \text { Bill }^{\prime} \text { s father }\right) \\
\lambda x \cdot \lambda w \cdot \text { call }_{w}\left(x, \text { Harold }^{\prime} \text { s father }\right) \\
\lambda x \cdot \lambda \text { call }_{w}\left(x, \text { Francis }^{\prime} \text { s father }\right)
\end{array}\right\}
$$

Now we notice that $\lambda y \cdot \lambda w\left[\operatorname{call}_{w}\left(y, y^{\prime}\right.\right.$ s father $\left.)\right](x) \notin\{g(C)\}(x)$ can only hold if the proposition $\lambda$ w.call ${ }_{w}\left(x, x^{\prime} s\right.$ father $)$ is not a member of the set resulting from applying each member of (32) to $x$, i.e., if it is not a member of the set of propositions in (33). In other words, the predicate in (31) is only defined for $x$ 's that are not identical to any of the students. It thus follows that (30) is ungrammatical. The quantifier every young student cannot be applied to the denotation of the VP as the latter is not defined for students.

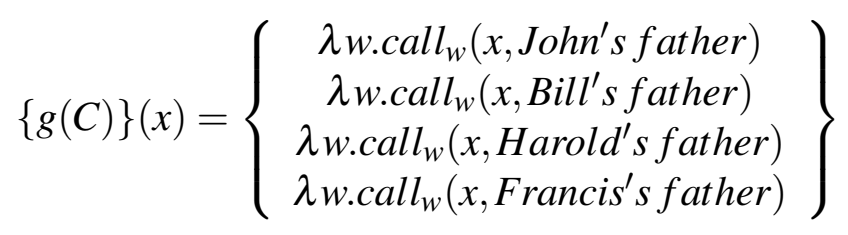

This means that the contrastiveness requirement of the $\sim$-operator not only explains why focus on a bound pronoun is obligatory if there is an embedded $\sim$-operator but, moreover, also why the domains of the quantifiers must not overlap in order for 
focus on the pronoun to be possible.

\subsection{Presence of too}

In section 2.2.3, I suggested that there are constructions that argue against the use of an E-type strategy for focused bound pronouns. The problem was the co-occurrence of focus on a bound variable and additive too as in (34), repeated from above.

Every director discussed his film, and every PRODUCER discussed HIS film, too

The problem for an E-type analysis is that the semantics of too, repeated in (35) from above, requires that replacing producer with director should result in a proposition that is true in the context given. But since the denotation of the VP, under the E-type analysis, is a partial function only defined for producers, this requirement cannot be satisfied. In other words, one ends up with an undefined truth value.

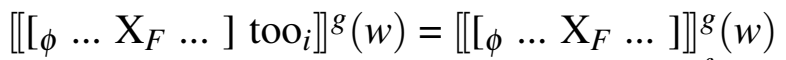

$$
\begin{aligned}
& \text { if } \left.\left[\left[\mathrm{Y}_{i}\right]\right]^{g} \in[[\mathrm{X}]]\right]^{f},\left[\left[\mathrm{Y}_{i}\right]\right]^{g} \neq[[\mathrm{X}]]^{g} \text { and } \\
& {\left[\left[\left[\begin{array}{lll}
\phi & \ldots & Y_{i} \ldots
\end{array}\right]\right]^{g}(w)=1\right. \text {, otherwise undefined }}
\end{aligned}
$$

As we have just seen in the previous subsection, the present proposal also makes use of partiality. But it is somewhat differently implemented. In the present theory the denotation of the VP in the second sentence of (34) is not only defined for producers. Rather it is only defined for those individuals that have the consequence that the predicate is not included in the denotation of $\mathrm{C}$ when both the former and the latter are applied to these individuals - that is, the proposition $\lambda$ w.discuss $_{w}\left(x, x^{\prime}\right.$ s film) must not be a member of $\{g(C)\}(x)$. When the quantifier every producer is applied to the predicate, it thus follows that $g(C)$ must be such that no producers serve as individuals introduced by the F-mark on the pronoun. In other words, if $g(C)$ is as in (36), then none of $a, b$, and $c$ must be a producer.

$$
g(C)=\left\{\begin{array}{l}
\lambda w_{1} \cdot \operatorname{discuss}_{w}\left(x, a^{\prime} s \text { film }\right) \\
\lambda \text { discuss }_{w}\left(x, b^{\prime} \text { s film }\right) \\
\lambda \text { didiscuss }_{w}\left(x, c^{\prime} s \text { film }\right)
\end{array}\right\}
$$

When the presupposition of too is checked, however, things change. What too requires, is that essentially the proposition in (37) is true in the context. I.e., it is again required that the proposition $\lambda w$.discuss $s_{w}\left(x, x^{\prime} s\right.$ film) must not be a member of $\{g(C)\}(x)$ - that is of (36). But since we are now applying the quantifier every director to the property denoted by the VP, the requirements change: $a, b$, and $c$ must not be directors. However, they could be producers. And that is exactly what 
Contrastive salient alternatives

the second sentence in (34) provides as salient alternatives that can serve as the denotation of $\mathrm{C}$.

$$
\begin{aligned}
& \lambda w . \forall x\left[\operatorname{director}_{w}(x) \rightarrow \lambda w^{\prime} \cdot \operatorname{discuss}_{w^{\prime}}\left(x, x^{\prime} s \text { film }\right) \notin\{g(C)\}(x) .\right. \\
& \left.\operatorname{discuss}_{w}\left(x, x^{\prime} \text { s film }\right)\right]
\end{aligned}
$$

In other words, because of the fact that the partial function does not directly state which individuals it is defined for, unlike the E-type account discussed, but rather does so by making use of a contextual set of alternatives, it turns out that the function is defined for differing individuals when used with one quantifier rather than with another. So the present proposal improves on previous analyses of focus on bound pronouns in a significant way.

\subsection{Paycheck pronouns}

Jacobson (2000) presents the contrast in (38) as an argument against an approach to focused bound pronouns making use of bound variables. Whereas the bound pronoun in (38-b) can be stressed contrastively, the paycheck pronoun in (38-a) cannot be stressed. This is surprising if paycheck pronouns have an underlying representation that is parallel to the DP in (38-b) - that is, if the paycheck pronoun her is to be constructed parallel to the DP his mother, where the antecedent DP provides the mother-of-function for the paycheck pronoun. The mother-of-function would then apply to an embedded bound variable (cf. Cooper 1979, Engdahl 1986 a.o.). ${ }^{13}$

a. *Every $3 d$ grade boy loves his mother, while every 4th grade boy HATES HER.

b. Every $3 \mathrm{~d}$ grade boy loves his mother, while every 4th grade boy HATES HIS mother.

(Jacobson 2000:(24),(25))

Jacobson notes that one cannot claim that AvoidF, the principle that strives to minimize focus, is responsible for the obligatory absence of focus on the paycheck pronoun in the grammatical version of (38-a). One could imagine that the focus on her is blocked because it is possible to stress less material than the whole DP, namely the bound variable embedded in her. Since the embedded bound variables contrast, one should stress them as this has the consequence that less material is F-marked than if the whole paycheck pronoun is F-marked. The problem is that this predicts that stress on the paycheck pronoun in (39) should be bad as well,

13 Alternatively, one could assume an ellipsis analysis for paycheck pronouns. Cf. Karttunen 1969, Partee 1975, Heim 1990, Elbourne 2005 a.o. 
contrary to fact. The generalization seems to be that only if there is a source for the underlying function of the paycheck pronoun applying to the bound pronoun other than the material that the paycheck pronoun is to be contrasted with, then F-marking the latter is possible.

Every $\operatorname{man}_{i}$ who loves his ${ }_{i}$ mother thinks that she ${ }_{f(i)}$ is nice, while every $\operatorname{man}_{j}$ who HATES HIS ${ }_{j}$ mother thinks that $\mathrm{SHE}_{f(j)}$ is a jerk. (Jacobson 2000:(28))

The problem is that the present approach can make the underlying bound pronouns contrast. I.e., it predicts (39) to be good. But then it is unclear why (38-a) is infelicitous. If we make the move to claim that the latter is unacceptable because too much material is F-marked, it becomes unclear why this is not so in the former case. ${ }^{14}$ This is a genuine and interesting problem. I would nevertheless claim that (38-a) is ruled out by AvoidF, i.e., too much material is stressed. This means that (39) is possible because more than the underlying bound pronouns contrast. In particular, the functions supplied in the first and the second conjunct in (39) differ: in the first conjunct it corresponds to the property of being the mother of $x$ and being loved by man $x$, whereas in the second conjunct it is the property of being the mother of $x$ and being hated by man $x$. These functions contrast. Therefore AvoidF dictates the stress on the whole paycheck pronoun. No such option is possible for (38-a). Here the paycheck pronoun is directly dependent on the DP his mother in the first conjunct. The details of such an analysis remain to be worked out, of course.

\section{Conclusion}

In the present paper I argued on the basis of novel data that an analysis without E-type pronouns is necessary for focus on bound pronouns. In other words, the cooccurrence of focus on bound pronouns and additive too suggests that an appropriate analysis must make use of F-marks on simple bound variables. We have seen that this in itself presents a challenge for the following reasons: first, traditional theories of focus licensing such as Rooth's (1992) do not readily allow for focus on bound variables. Second, the optionality of focus on bound pronouns is confusing because the construction without F-mark should block the one with (Schwarzschild 1999). I devised an essentially Roothian theory of focus licensing that is able to deal with these challenges. In particular, I argued that $\sim$-operators must be inserted in the scope of the quantifier when a bound pronoun bears contrastive stress.

14 A parallel problem arises in Sauerland's (2008) theory because here it is also the bound pronoun inside the paycheck pronoun that is supposed to contrast with an antecedent pronoun. In Sauerland's case it would be an E-type pronoun with a bound variable in it that the function applies to. Crucially, though, the paycheck pronoun is also complex in his case. 
Contrastive salient alternatives

This allowed us to deal with the apparent optionality of the F-mark by making the optionality follow from a syntactic difference between the option with focus and the one without. Moreover, I introduced the novel concept of salient alternative whereby the alternatives that are used for the contextual restriction of the $\sim$-operator can be compositionally reconstructed. This had the consequence that focus on bound pronouns can be licensed at all. Finally, a modification of the $\sim$-operator was suggested which incorporates contrastiveness in the lexical entry of the $\sim$-operator. This modification makes focus on bound pronouns obligatory if the evaluating operator is embedded in the scope of the quantifier. The resulting theory was shown to be able to deal with the problematic facts with additive too, whereas previous theories were shown to be unable to do so.

\section{References}

Chierchia, Gennaro. 1990. Anaphora and dynamic binding. Linguistics and Philosophy 15. 111-183.

Cooper, Robin. 1979. The interpretation of pronouns. In Frank Heny \& H. Schnelle (eds.), Syntax and semantics 10: Selections from the third Groningen round table, 61-92. New York: Academic Press.

Dimitriadis, Alexis. 2001. Function domains in variable free semantics. In Rachel Hastings, Brendan Jackson \& Zsofia Zvolenszky (eds.), Proceedings of SALT 11, 134-151. Cornell University, Ithaca, NY: CLC Publications.

Elbourne, Paul D. 2005. Situations and individuals. MIT Press.

Engdahl, Elisabet. 1986. Constituent questions. Dordrecht, The Netherlands: D. Reidel Publishing Company.

Hamblin, Charles. 1973. Questions in Montague grammar. Foundations of Language 10(1). 41-53.

Heim, Irene. 1990. E-type pronouns and donkey anaphora. Linguistics and Philosophy 13(2). 137-178.

Heim, Irene. 1992. Presupposition projection and the semantics of attitude verbs. Journal of Semantics 9. 183-221.

Heim, Irene \& Angelika Kratzer. 1998. Semantics in generative grammar. Malden, MA: Blackwell.

Jacobson, Pauline. 2000. Paychecks, stress, and variable free semantics. In T. Matthews \& B. Jackson (eds.), Proceedings of SALT X, 65-82. Cornell University, Ithaca, NY: CLC Publications.

Kaplan, Jeff. 1984. Obligatory too in English. Language 60(3). 510-518. doi:10.2307/413989.

Karttunen, Lauri. 1969. Pronouns and variables. In Robert I. Binnick (ed.), Proceed- 
ings of the fifth regional meeting of the Chicago Linguistics Society, 108-116. Chicago: Chicago Linguistics Society.

Mayr, Clemens. 2010. The role of alternatives and strength in grammar. Cambridge, Massachusetts: Harvard University dissertation.

Partee, Barbara. 1975. Deletion and variable binding. In Edward L. Keenan (ed.), Foral semantics of natural language, Cambridge: Cambridge University Press.

Rooth, Mats. 1985. Association with focus. University of Massachusetts, Amherst: University of Massachusetts, Amherst dissertation.

Rooth, Mats. 1992. A theory of focus interpretation. Natural Language Semantics 1(1). 75-116. doi:10.1007/BF02342617.

Sauerland, Uli. 1998. The meaning of chains. Cambridge, Massachusetts: Massachusetts Institute of Technology dissertation.

Sauerland, Uli. 2000. The content of pronouns: Evidence from focus. In Brendan Jackson \& Tanya Matthews (eds.), Proceedings of SALT X, 167-184. Cornell University, Ithaca, NY: CLC Publications.

Sauerland, Uli. 2008. The silent content of bound variable pronouns. In Kyle Johnson (ed.), Topics in ellipsis, 183-209. Cambridge, United Kingdom: Cambridge University Press.

Schwarzschild, Roger. 1999. Givenness, AvoidF and other constraints on the placement of accent. Natural Language Semantics 7(2). 141-177. doi:10.1023/A:1008370902407.

Clemens Mayr

Zentrum für Allgemeine Sprachwissenschaft

Schützenstraße 18

D-10117 Berlin

mayr@zas.gwz-berlin.de 